Pacific Journal of Mathematics

LIOUVILLE'S THEOREM ON FUNCTIONS WITH 


\title{
LIOUVILLE'S THEOREM ON FUNCTIONS WITH ELEMENTARY INTEGRALS
}

\author{
MAXWELL RosenLICHT
}

\begin{abstract}
Defining a function of one variable to be elementary if it has an explicit representation in terms of a finite number of algebraic operations, logarithms, and exponentials, Liouville's theorem in its simplest case says that if an algebraic function has an elementary integral then the latter is itself an algebraic function plus a sum of constant multiples of logarithms of algebraic functions. Ostrowski has generalized Liouville's results to wider classes of meromorphic functions on regions of the complex plane and J. F. Ritt has given the classical account of the entire subject in his Integration in Finite Terms, Columbia University Press, 1948. In spite of the essentially algebraic nature of the problem, all proofs so far have been analytic. This paper gives a self contained purely algebraic exposition of the problem, making a few new points in addition to the resulting simplicity and generalization.
\end{abstract}

A differential ring is a commutative ring $R$ together with a derivation of $R$ into itself, that is, a map $R \rightarrow R$ which, if denoted $x \mapsto x^{\prime}$, satisfies the two rules

$$
\begin{aligned}
& (x+y)^{\prime}=x^{\prime}+y^{\prime} \\
& (x y)^{\prime}=x^{\prime} y+x y^{\prime} .
\end{aligned}
$$

A differential field is a differential ring that is a field.

If $u, v$ are elements of a differential field and $v \neq 0$ we have the relation $(u / v)^{\prime}=\left(u^{\prime} v-u v^{\prime}\right) / v^{2}$. In a differential ring we have $\left(x^{n}\right)^{\prime}=$ $n x^{n-1} x^{\prime}$ for $n=1,2,3, \cdots$. In particular, setting $x=1, n=2$, we have $1^{\prime}=0$. Elements of derivative zero are called constants, and in a differential field the totality of constants is itself a field, the subfield of constants.

If $u, v$ are elements of a differential field such that $v \neq 0$ and $u^{\prime}=v^{\prime} / v$, in analogy with the classical situation we say that $u$ is a logarithm of $v$ or that $v$ is an exponential of $u$. If, in a certain differential field, $v$ has a logarithm, then it is necessarily unique to within an additive constant, while if $u$ has an exponential, the latter is necessarily unique to within multiplication by a nonzero constant. A differential extension field of a differential field is said to be elementary if there exists a finite tower of intermediate differential fields, starting with the given small field and ending with the given extension field, such that each field in the tower after the first is 
obtained from its predecessor by the adjunction of a single element that is a logarithm or an exponential of an element of the preceding field or algebraic over the proceding field. If we are dealing with fields of meromorphic functions on a given region of the plane (or sphere) of the complex variable $z$, differentiation being the ordinary $d / d z$, then elements of elementary differential extension fields of the differential field $\boldsymbol{C}(z)$ of rational functions of $z$ are called elementary functions. For example, the trigonometric and inverse trigonometric functions of $z$ are elementary functions of $z$, and so are composites of elementary functions of $z$, all on suitable domains of definition in $C$ or on Riemann surfaces.

For the convenience of the reader we include a proof of the following well-known result.

Proposition. Let $F$ be a differential field of characteristic zero and $K$ an extension field of $F$. Then there exists a differential field structure on $K$ that is compatible with that of $F$ and with the field structure of $K$. This differential field structure on $K$ is unique if $K$ is algebraic over $F$ and in any case induces a differential field structure on any subfield of $K$ that contains and is algebraic over $F$.

We have first of all to show that a derivation $D$ on $F$ can be extended to one on $K$. If $K=F(X)$, with $X$ transcendental over $F$, then the map

$$
D_{0}: F[X] \longrightarrow F[X]
$$

defined by

$$
D_{0}\left(\sum_{i=0}^{n} a_{i} X^{i}\right)=\sum_{i=0}^{n}\left(D a_{i}\right) X^{i}
$$

if $a_{0}, a_{1}, \cdots, a_{n} \in F$, is a derivation of $F[X]$ extending $D . \quad D_{0}$ can be extended to a derivation of the quotient field $K=F(X)$ of $F[X]$ by setting, for $u, v \in F[X], v \neq 0$,

$$
D_{0}(u / v)=\left(\left(D_{0} u\right) v-\left(D_{0} v\right) u\right) / v^{2} .
$$

Next suppose that $K=F(x)$, with $x$ algebraic over $F$. Let $X$ be an indeterminate over $F$ and let $f(X) \in F[X]$ be the minimal polynomial of $x$ over $F$. The map

$$
\partial / \partial X: F[X] \longrightarrow F[X]
$$

defined by

$$
\partial / \partial X\left(\sum_{i=0}^{n} a_{i} X^{i}\right)=\sum_{i=0}^{n} i a_{i} X^{i-1}
$$


if $a_{0}, a_{1}, \cdots, a_{n} \in F$, is a derivation of $F[X]$ that annuls each element of $F$. Thus for any $g(X) \in F[X]$ the additive map $D_{0}+g(X) \partial / \partial X$ is a derivation of $F[X]$ that extends $D$. Setting $f^{\prime}(X)=(\partial / \partial X) f$, we have $f^{\prime}(x) \neq 0$, and since $F(x)=F[x]$ we can find a particular $g(X) \in F[X]$ such that

$$
\left(D_{0} f\right)(x)+g(x) f^{\prime}(x)=0 .
$$

Then $D_{0}+g(X) \partial / \partial X$ maps $f(X)$ into a multiple of itself, hence maps the ideal $F[X] f(X)$ of $F[X]$ into itself, hence induces a derivation on the factor ring $F[X] / F[X] f(X)$, which is isomorphic to $F(x)$. This gives us the desired extension of $D$ to $K=F(x)$. Thus $D$ can be extended to a derivation of any simple extension field of $F$. That $D$ can be extended to a derivation of an arbitrary extension field of $F$ is an immediate consequence of Zorn's lemma. To complete the proof it suffices to show that if $D_{1}$ and $D_{2}$ are two derivations of the field $K$ that agree on the subfield $F$ and $x \in K$ is algebraic over $F$ then $D_{1} x=D_{2} x$. Passing to the derivation $D_{1}-D_{2}$ of $K$, we have to show that any derivation of $K$ which annuls all of $F$ also annuls each $x \in K$ that is algebraic over $F$. This is done by noting that if $f(X) \in F[X]$ is the minimal polynomial of $x$ over $F$ then we have $0=(f(x))^{\prime}=f^{\prime}(x) \cdot x^{\prime}$, so that $x^{\prime}=0$.

The following result is the meat of this paper.

Lemma. Let $F$ be a differential field of characteristic zero, $F(t)$ a differential extension field of $F$ with the same field of constants and with $t$ transcendental over $F$ and either $t^{\prime} \in F$ or $t^{\prime} / t \in F$. Let $c_{1}, \cdots, c_{n} \in F$ be linearly independent over the rational numbers $Q$ and let $u_{1}, \cdots, u_{n}$ be nonzero elements of $F(t), v \in F(t)$. Then if

$$
\sum_{i=1}^{n} c_{i} \frac{u_{i}^{\prime}}{u_{i}}+v^{\prime} \in F[t]
$$

we have $v \in F[t]$ and, in the case $t^{\prime} \in F$, each $u_{i} \in F$, while in the case $t^{\prime} / t \in F$, for each $i=1, \cdots, n$ we have $u_{i} / t^{\nu} i \in F$ for some integer $\nu_{i}$.

In a suitable finite normal algebraic extension field $K$ of $F$ the quantities $u_{1}, \cdots, u_{n}, v$ will split into linear factors, so that we can write

$$
\begin{aligned}
& u_{i}=g_{i} \prod_{j}\left(t-z_{j}\right)^{\mu_{i j}}, \quad i=1, \cdots, n \\
& v=\sum_{j, \nu} h_{\nu j}\left(t-z_{j}\right)^{\nu}+(\text { element of } K[t]),
\end{aligned}
$$

where $j$ ranges over a finite set of positive integers, $\nu$ ranges over a 
finite set of negative integers, each $\mu_{i j}$ is an integer, and each $g_{i}, z_{j}, h_{\nu j} \in K$. Of course each $g_{i}$ is nonzero. We work in the differential extension field $K(t)$ of $F(t) . \quad K$ is a differential subfield, $K[t]$ a differential subring of $K(t)$. By assumption we have

$$
\sum_{i=1}^{n} c_{i} \frac{g_{i}^{\prime}}{g_{i}}+\sum_{i, j} c_{i} \mu_{i j} \frac{t^{\prime}-z_{j}^{\prime}}{t-z_{j}}+\sum_{j, \nu}\left(h_{\nu j}\left(t-z_{j}\right)^{\nu}\right)^{\prime} \in K[t] .
$$

The idea of the following proof is, roughly speaking, that when we express the various functions appearing here as quotients of polynominals in $t$ we get no cancellation, and when we bring the various terms together we still get no cancellation, so that all the $h_{\nu j}$ 's and all the $\mu_{i j}$ 's (except those corresponding to $z_{j}=0$ ) vanish.

We have either $t^{\prime}=a$ or $t^{\prime}=a t$, for some $a \in F$. In the former case,

$$
\frac{t^{\prime}-z_{j}^{\prime}}{t-z_{j}}=\frac{a-z_{j}^{\prime}}{t-z_{j}}
$$

Here $a-z_{j}^{\prime} \in K$, and we claim that the last fraction is in lowest terms; that is, we claim that $a-z_{j}^{\prime} \neq 0$. To see this, note that by the unicity part of the proposition, differentiation commutes with each $F$-automorphism of $K$, so that if $a=z_{j}^{\prime}$ then for each $\sigma \in \operatorname{Aut}(K / F)$ we have $a=\left(\sigma z_{j}\right)^{\prime}$, so that $[K: F] a=\left(\sum_{\sigma} \sigma z_{j}\right)^{\prime}$. Hence $a=b^{\prime}$, for some $b \in F$, giving $(t-b)^{\prime}=0$, or $t-b$ a constant. Since each constant of $F(t)$ is in $F$, we get $t \in F$, a contradiction. Thus $a-z_{j}^{\prime} \neq 0$. In the case $t^{\prime}=a t$ we have

$$
\frac{t^{\prime}-z_{i}^{\prime}}{t-z_{j}}=\frac{a t-z_{i}^{\prime}}{t-z_{j}},
$$

and we claim that also this fraction is in lowest terms, provided $z_{j} \neq 0$. For if the fraction were not in lowest terms we would have $z_{j}^{\prime}=a z_{j}$, so $z_{j}^{\prime} / z_{j}=a$, so $\left(\sigma z_{j}\right)^{\prime} / \sigma z_{j}=a$ for each $\sigma \in \operatorname{Aut}(K / F)$. Therefore

$$
[K: F] a=\sum_{\sigma} \frac{\left(\sigma z_{j}\right)^{\prime}}{\sigma z_{j}}=\left(\prod_{\sigma} \sigma z_{j}\right)^{\prime} / \prod_{\sigma} \sigma z_{j}
$$

Thus $N a=b^{\prime} / b$, where $N=\left[K: F^{\prime}\right]$ and $b \in F, b \neq 0$. Hence

$$
\left(t^{N}\right)^{\prime} / t^{N}=N t^{\prime} / t=N a=b^{\prime} / b,
$$

giving $\left(t^{N} / b\right)^{\prime}=0$, so $t^{N} / b \in F$, a contradiction. Thus in all cases but one, the fraction $\left(t^{\prime}-z_{j}^{\prime}\right) /\left(t-z_{j}\right)$ is in lowest terms, a nonzero linear expression in $t$ divided by $t-z_{j}$; the exceptional case occurs when $t^{\prime} / t \in F$ and $z_{j}=0$, where $\left(t^{\prime}-z_{j}^{\prime}\right) /\left(t-z_{j}\right)=t^{\prime} / t \in F$. 
Now

$$
\left(h_{\nu j}\left(t-z_{j}\right)^{\nu}\right)^{\prime}=h_{\nu j}^{\prime}\left(t-z_{j}\right)^{\nu}+\nu h_{\nu j}\left(t-z_{j}\right)^{\nu-1}\left(t^{\prime}-z_{j}^{\prime}\right) .
$$

By what has been done above, except in the one exceptional case, if $h_{\nu j} \neq 0$ we have here an expression which in lowest terms has a nonzero linear numerator and a denominator $\left(t-z_{j}\right)^{-\nu+1}$ (of degree $-\nu+1>1$ ). In the exceptional case $t^{\prime} / t=a \in F, z_{j}=0$, we have

$$
\left(h_{\nu j}\left(t-z_{j}\right)^{\nu}\right)^{\prime}=t^{\nu}\left(h_{\nu j}^{\prime}+\nu h_{\nu j} a\right),
$$

and we claim that if $h_{\nu j} \neq 0$ then also $h_{\nu j}^{\prime}+\nu h_{\nu j} a \neq 0$. For from the equation $h_{\nu j}^{\prime} / h_{\nu j}=-\nu a$ we would get $\left(\sigma h_{\nu j}\right)^{\prime} /\left(\sigma h_{\nu j}\right)=-\nu a$ for each $\sigma \in \operatorname{Aut}(K / F)$, and hence

$$
-[K: F] \nu a=\sum_{\sigma}\left(\sigma h_{\nu j}\right)^{\prime} /\left(\sigma h_{\nu j}\right)=\left(\prod_{\sigma} \sigma h_{\nu j}\right)^{\prime} / \prod_{\sigma} \sigma h_{\nu j},
$$

or $-N \nu a=b^{\prime} / b$, with $N=[K: F]$ and $b \in F, b \neq 0$, so that

$$
\left(t^{-N \nu}\right)^{\prime} /\left(t^{-N \nu}\right)=-N \nu a=b^{\prime} / b,
$$

so that $\left(t^{-N \nu} / b\right)^{\prime}=0$, giving, as before, $t^{-N^{\nu}} / b \in F$, a contradiction. Thus in the exceptional case $\left(h_{\nu j}\left(t-z_{j}\right)^{\nu}\right)^{\prime}$ in lowest terms has denominator $t^{-\nu}$ (of degree $-\nu>0$ ). If now we had some $h_{\nu j} \neq 0$, then the various terms of the left-hand side of $\left({ }^{*}\right)$ would not cancel, and they could not possibly add up to a polynomial in $t$. Thus all $h_{\nu j}$ 's are zero, giving $v \in K[t]$. Hence $v \in K[t] \cap F(t)=F[t]$.

We are left with

$$
\sum_{i, j} c_{i} \mu_{i j} \frac{t^{\prime}-z_{j}^{\prime}}{t-z_{j}} \in K[t] .
$$

We have seen that, except in the exceptional case, each $\left(t^{\prime}-z_{j}^{\prime}\right) /\left(t-z_{j}\right)$ is already in lowest terms, a fraction with denominator $t-z_{j}$. For cancellation to take place we must have each $\sum_{i} c_{i} \mu_{i j}=0$. By the linear independence of $c_{1}, \cdots, c_{n}$ over $Q$ we must have each $\mu_{i j}=0$. Allowing for the exceptional case, there are integers $\nu_{1}, \cdots, \nu_{n}$ (all zero in case $t^{\prime} \in F$, and equal to the integer $\mu_{i j}$ corresponding to $z_{j}=0$ in the case $\left.t^{\prime} / t \in F\right)$ such that $u_{i} / t^{\nu i} \in K$. Hence $u_{i} / t^{\nu i} \in K \cap F(t)=F$. This completes the proof of the lemma.

The classical result of Liouville and its generalization by Ostrowski are contained in the following statement.

THEoREM. Let $F$ be a differential field of characteristic zero, $\alpha \in F$. If the equation $y^{\prime}=\alpha$ has a solution in some elementary differential extension field of $F$ having the same subfield of constants, then there exist constants $c_{1}, \cdots, c_{n} \in F$ and elements $u_{1}, \cdots, u_{n}, v \in F$ 
such that

$$
\alpha=\sum_{i=1}^{n} c_{i} \frac{u_{i}^{\prime}}{u_{i}}+v^{\prime} .
$$

By assumption there is a tower of differential fields

$$
F=F_{0} \subset F_{1} \subset \cdots \subset F_{N},
$$

all having the same constant field, such that $\alpha \in F_{N}^{\prime}$ and for each $i=1, \cdots, N, F_{i}=F_{i-1}\left(t_{i}\right)$, where $t_{i}$ is a logarithm or an exponential of an element of $F_{i-1}$, or algebraic over $F_{i-1}$. The proof is by induction on $N$, the case $N=0$ being trivial. If $N>0$, we can apply the induction assumption to the same $\alpha$ and the tower $F_{1} \subset F_{2} \subset \cdots \subset F_{N}$ to get

$$
\alpha=\sum_{i=1}^{n} c_{i} \frac{u_{i}^{\prime}}{u_{i}}+v^{\prime},
$$

where now $c_{1}, \cdots, c_{n}$ are, as desired, constants in $F$, but $u_{1}, \cdots, u_{n}, v$ are in $F_{1}$. Writing $t_{1}=t$, we have $F_{1}=F(t)$, where $t$ is a logarithm or an exponential of an element of $F$, or algebraic over $F$, and the above equation holds with constants $c_{1}, \cdots, c_{n}$ and elements $u_{1}, \cdots, u_{n}, v$ in $F(t)$. We want to modify $n, c_{1}, \cdots, c_{n}, u_{1}, \cdots, u_{n}, v$ in such a manner that the same equation holds and we have the stronger relations $u_{1}, \cdots, u_{n}, v \in F$. First suppose that $c_{1}, \cdots, c_{n}$ are linearly dependent over $Q$, for example that we have a relation

$$
c_{n}=\left(m_{1} c_{1}+\cdots+m_{n-1} c_{n-1}\right) / m,
$$

with $m_{1}, \cdots, m_{n-1}, m$ integers and $m \neq 0$. For $i=1, \cdots, n-1$ we can replace each $u_{i}$ by $u_{i}^{m}$ and $c_{i}$ by $c_{i} / m$ to get the same situation as before, but $c_{n}=m_{1} c_{1}+\cdots+m_{n-1} c_{n-1}$. Thus

$$
\begin{aligned}
\alpha & =c_{1}\left(u_{1}^{\prime} / u_{1}+m_{1} u_{n}^{\prime} / u_{n}\right)+\cdots+c_{n-1}\left(u_{n-1}^{\prime} / u_{n-1}+m_{n-1} u_{n}^{\prime} / u_{n}\right)+v^{\prime} \\
& =c_{1}\left(u_{1} u_{n}^{m_{1}}\right)^{\prime} /\left(u_{1} u_{n}^{m_{1}}\right)+\cdots+c_{n-1}\left(u_{n-1} u_{n}^{m_{n-1}}\right)^{\prime} /\left(u_{n-1} u_{n}^{m_{n-1}}\right)+v^{\prime},
\end{aligned}
$$

and we have the same situation as before with smaller $n$. Thus we may suppose that $c_{1}, c_{2}, \cdots, c_{n}$ are linearly independent over $Q$. If $t$ is a logarithm of an element of $F$ we have $t^{\prime}=a^{\prime} / a$, for some $a \in F$. If $t$ is transcendental over $F$ the lemma is applicable, and we get that each $u_{i} \in F$, while $v \in F[t]$. We also have $v^{\prime} \in F$, and we shall be done in the present case if we can show that $v \in F[t], v^{\prime} \in F$ together imply that $v=c t+d$, for some constant $c \in F$ and some element $d \in F$. To do this, write

$$
v=\sum_{j=0}^{m} b_{j} t^{j}
$$


where each $b_{j} \in F$ and assume, as we may, that $m>0, b_{m} \neq 0$. Then

$$
\begin{aligned}
v^{\prime}= & b_{m}^{\prime} t^{m}+\left(m b_{m} \frac{a^{\prime}}{a}+b_{m-1}^{\prime}\right) t^{m-1} \\
& +(\text { element of } F[t] \text { of degree }<m-1) .
\end{aligned}
$$

Since $v^{\prime} \in F$, we get $b_{m}^{\prime}=0$, so $b_{m}$ is a constant, and if $m>1$ we get $m b_{m} a^{\prime} / a+b_{m-1}^{\prime}=0$, that is $\left(m b_{m} t+b_{m-1}\right)^{\prime}=0$, so that $m b_{m} t+b_{m-1} \in F$, contradicting the transcendency of $t$ over $F$; thus $m=1$ and the present case is done. In the next case $t$ is an exponential of an element of $F$, so that we have $t^{\prime} / t=a^{\prime}$, for some $a \in F$. If $t$ is transcendental over $F$, the lemma says that each $u_{i}$ is of the form $u_{i}=$ $a_{i} t^{\nu i}$, with $a_{i} \in F$ and $\nu_{i}$ an integer, and that $v \in F[t]$. Since

$$
u_{i}^{\prime} / u_{i}=a_{i}^{\prime} / a_{i}+\nu_{i} t^{\prime} / t=a_{i}^{\prime} / a_{i}+\nu_{i} a^{\prime}
$$

we have

$$
\alpha=\sum_{i=1}^{n} c_{i} \frac{a_{i}^{\prime}}{a_{i}}+\left(v+\left(\nu_{1}+\cdots+\nu_{n}\right) a\right)^{\prime},
$$

so that we can assume that each $u_{i} \in F$. Then $v^{\prime} \in F$, and it will suffice to show that $v \in F$. Again writing

$$
v=\sum_{j=0}^{m} b_{j} t^{j}
$$

with each $b_{j} \in F$ and $b_{m} \neq 0$, we have

$$
v^{\prime}=\sum_{j=0}^{m}\left(b_{j}^{\prime}+j b_{j} a^{\prime}\right) t^{j} .
$$

If $m \neq 0$ we have $b_{m}^{\prime}+m b_{m} a^{\prime}=0$, so that $b_{m}^{\prime} / b_{m}+m t^{\prime} / t=0$, or $\left(b_{m} t^{m}\right)^{\prime}=0$, giving $b_{m} t^{m} \in F$, which is impossible. This disposes of all cases where $F_{1}=F(t)$ is transcendental over $F$. In the final case, $F_{1}$ algebraic over $F$, let $K$ be the smallest normal algebraic extension field of $F$ that contains $F_{1}$. For any $\sigma \in \operatorname{Aut}(K / F)$ we have

$$
\alpha=\sum_{i=1}^{n} c_{i} \frac{\left(\sigma u_{i}\right)^{\prime}}{\sigma u_{i}}+(\sigma v)^{\prime},
$$

so that

$$
[K: F] \alpha=\sum_{i=1}^{n} c_{i} \sum_{\sigma} \frac{\left(\sigma u_{i}\right)^{\prime}}{\sigma u_{i}}+\sum_{\sigma}(\sigma v)^{\prime}=\sum_{i=1}^{n} c_{i} \frac{\left(\prod_{\sigma} \sigma u_{i}\right)^{\prime}}{\prod_{\sigma} \sigma u_{i}}+\left(\sum_{\sigma} \sigma v\right)^{\prime} .
$$

Dividing both sides by $[K: F]$ and noting that $\Pi_{\sigma} \sigma u_{i}$ and $\sum_{\sigma} \sigma v$ are in $F$ completes the proof. 
EXAMPLE. As a further illustration of the utility of the lemma, let us consider which functions $f(z) e^{g(z)}$ of a complex variable $z$ have elementary integrals, if $f$ and $g$ are rational functions of $z, f$ being nonzero and $g$ being nonconstant. Writing $e^{g}=t$, we start with the differential field $\boldsymbol{C}(z, t)$, in which $C$ is the constant field and $z^{\prime}=1$, $t^{\prime}=g^{\prime} t$. If $f e^{g}$ has an elementary integral then we can write

$$
f t=\sum_{i=1}^{n} c_{i} \frac{u_{i}^{\prime}}{u_{i}}+v^{\prime}
$$

with $c_{1}, \cdots, c_{n} \in \boldsymbol{C}$, and $u_{1}, \cdots, u_{n}, v \in \boldsymbol{C}(z, t)$. Now $t$ is transcendental over $C(z)$, as can be seen either analytically, or also algebraically, noting that if $t$ is contained in a finite normal algebraic extension field $F$ of $\boldsymbol{C}(z)$ then $(\sigma t)^{\prime} / \sigma t=g^{\prime}$ for each $\sigma \in \operatorname{Aut}(F / C(z))$, so

$$
[F: C(z)] g^{\prime}=\sum_{\sigma}\left((\sigma t)^{\prime} / \sigma t\right)=\left(\prod_{\sigma} \sigma t\right)^{\prime} / \prod_{\sigma} \sigma t,
$$

so that for some nonconstant $u \in C(z)$ we have $u^{\prime} / u$ the derivative of an element of $C(z)$, contrary to the fact that all the poles of $u^{\prime} / u$ are of order one. Assuming, as we may (by an argument occurring in the proof of the theorem), that $c_{1}, \cdots, c_{n}$ are linearly independent over $Q$, we apply the lemma to the differential fields $C(z) \subset \boldsymbol{C}(z, t)$, getting that $v \in \boldsymbol{C}(z)[t]$ and each $u_{i}$ is the product of an element of $\boldsymbol{C}(z)$ with a power of $t$. Hence

$$
f t=v^{\prime}+(\text { element of } C(z)),
$$

with $v \in \boldsymbol{C}(z)[t]$. Writing

$$
v=\sum_{j=0}^{m} b_{j} t^{j},
$$

with $b_{0}, \cdots, b_{m} \in \boldsymbol{C}(z), b_{m} \neq 0$, we get

$$
v^{\prime}=\sum_{j=0}^{m}\left(b_{j}^{\prime}+j b_{j} g^{\prime}\right) t^{j}
$$

If $j, b_{j} \neq 0$, we also have $b_{j}^{\prime}+j b_{j} g^{\prime} \neq 0$, since otherwise $g^{\prime}$ would have all its poles of order one, which is imposible. Thus $m=1$, and setting $b_{1}=a$ we get $f=a^{\prime}+a g^{\prime}$. Conversely if there exists an $a \in C(z)$ such that $f=a^{\prime}+a g^{\prime}$ then $f e^{g}$ has an elementary integral, namely $a e^{g}$. Thus we get the necessary and sufficient condition, due to Liouville himself, that $\int f e^{g}$ be elementary, if $f, g \in \boldsymbol{C}(z), f \neq 0$, $g \notin C$ : there must exist $a \in C(z)$ such that $f=a^{\prime}+a g^{\prime}$. The same result holds, with the same argument, if $C(z)$ is replaced by any algebraic extension field of $\boldsymbol{C}(z)$. 
The classic integrals $\int e^{z^{2}} d z, \int\left(e^{z} / z\right) d z$ are not elementary, for they give rise to the equations $1=a^{\prime}+2 z a$ and $1 / z=a^{\prime}+a$, neither of which has a solution $a \in C(z)$, as one sees by looking at the partial fraction expression for $a$.

Received February 28, 1967. Research supported by National Science Foundation grant number GT-3990.

UNIVERSity of CALIForNia, BerkeLey 



\section{PACIFIC JOURNAL OF MATHEMATICS}

\section{EDITORS}

\section{H. ROYDEN}

Stanford University

Stanford, California

\section{J. P. Jans}

University of Washington

Seattle, Washington 98105

\section{J. DUGUNDJI}

Department of Mathematics

Rice University

Houston, Texas 77001

RICHARD ARENS

University of California

Los Angeles, California 90024

\section{ASSOCIATE EDITORS}
E. F. BeCKenbaCH
B. H. NeumanN
F. WOLF
K. YosIDA

\section{SUPPORTING INSTITUTIONS}

UNIVERSITY OF BRITISH COLUMBIA CALIFORNIA INSTITUTE OF TECHNOLOGY UNIVERSITY OF CALIFORNIA MONTANA STATE UNIVERSITY UNIVERSITY OF NEVADA NEW MEXICO STATE UNIVERSITY OREGON STATE UNIVERSITY UNIVERSITY OF OREGON OSAKA UNIVERSITY UNIVERSITY OF SOUTHERN CALIFORNIA
STANFORD UNIVERSITY UNIVERSITY OF TOKYO UNIVERSITY OF UTAH WASHINGTON STATE UNIVERSITY UNIVERSITY OF WASHINGTON

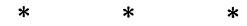

AMERICAN MATHEMATICAL SOCIETY CHEVRON RESEARCH CORPORATION TRW SYSTEMS

NAVAL ORDNANCE TEST STATION 


\section{Pacific Journal of Mathematics}

\section{Vol. 24, No. $1 \quad$ May, 1968}

Harry P. Allen, Lie algebras of type $D_{4}$ over algebraic number fields ...... 1

Charles Ballantine, Products of positive definite matrices. II............ 7

David W. Boyd, The spectral radius of averaging operators ............ 19

William Howard Caldwell, Hypercyclic rings ................... 29

Francis William Carroll, Some properties of sequences, with an application

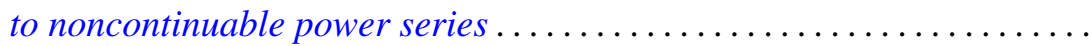

David Fleming Dawson, Matrix summability over certain classes of

sequences ordered with respect to rate of convergence ........... 51

D. W. Dubois, Second note on David Harrison's theory of preprimes. . . . . 57

Edgar Earle Enochs, A note on quasi-Frobenius rings.............. 69

Ronald J. Ensey, Isomorphism invariants for Abelian groups modulo bounded groups ................................ 71

Ronald Owen Fulp, Generalized semigroup kernels ................ 93

Bernard Robert Kripke and Richard Bruce Holmes, Interposition and approximation ................................. 103

Jack W. Macki and James Sai-Wing Wong, Oscillation of solutions to second-order nonlinear differential equations ..................

Lothrop Mittenthal, Operator valued analytic functions and generalizations

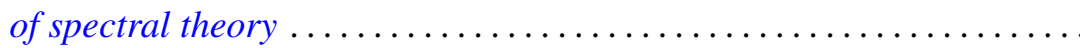

T. S. Motzkin and J. L. Walsh, A persistent local maximum of the pth power deviation on an interval, $p<1 \ldots \ldots \ldots \ldots \ldots \ldots \ldots \ldots \ldots \ldots . \ldots \ldots$

Jerome L. Paul, Sequences of homeomorphisms which converge to homeomorphisms ...........................

Maxwell Alexander Rosenlicht, Liouville's theorem on functions with elementary integrals.

Joseph Goeffrey Rosenstein, Initial segments of degrees .

$\mathrm{H}$. Subramanian, Ideal neighbourhoods in a ring ............

Dalton Tarwater, Galois cohomology of abelian groups . .

James Patrick Williams, Schwarz norms for operators ... .

Raymond Y. T. Wong, A wild Cantor set in the Hilbert cube. 